\title{
Noninvasive visualization of obesity-boosted inflammation in orthotopic pancreatic ductal adenocarcinoma using an octapod iron oxide nanoparticle
}

Tingting $\mathrm{Xu}^{1^{\dagger}}$, Xiaoxuan $\mathrm{Xu}^{1^{\dagger}}$, Lijiao Yang ${ }^{2}$, Xiaoyuan Chen ${ }^{2}$, Shenghong $\mathrm{Ju}^{* 1}$

${ }^{1}$ Jiangsu Key Laboratory of Molecular and Functional Imaging, Department of Radiology, Zhongda Hospital, Medical School of Southeast University, Nanjing, China.

${ }^{2}$ Laboratory of Molecular Imaging and Nanomedicine, National Institute of Biomedical Imaging and Bioengineering, National Institutes of Health, Bethesda, Maryland 20892, USA.

${ }^{\dagger}$ These authors contributed equally to this work.

* Corresponding Author: Shenghong Ju, Jiangsu Key Laboratory of Molecular and Functional Imaging, Department of Radiology, Zhongda Hospital, Medical School of Southeast University, 210009 Nanjing, China. E-mail: jsh0836@hotmail.com. 
Figure S1. Body weight of obese $(o b / o b)$ and lean WT mice ( $n=6$ per group). The data are reported as the mean $\pm \mathrm{SD}$.

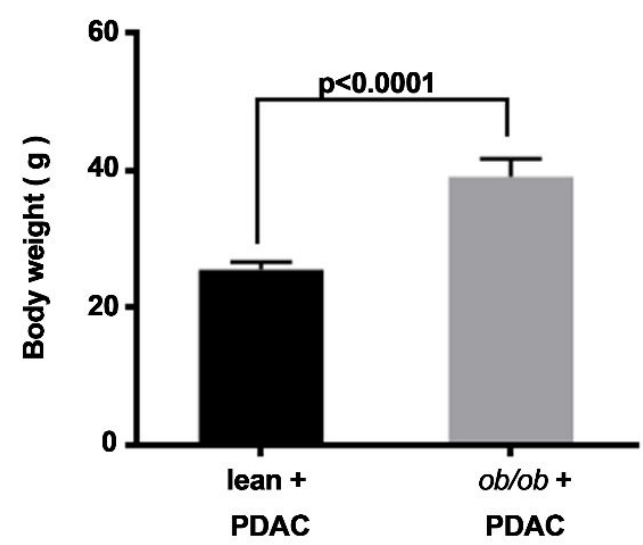


Figure S2. The tumour volume in lean and obese mice was monitored using $T_{2}$-weighted MR imaging. Tumour length and width were measured manually in the largest cross-sectional area of $T_{2}$-weighted imaging and the tumour volume was calculated according to the formula $\mathrm{V}=\left(\right.$ width $^{2} \times$ length $) / 2$. Data are reported as the mean \pm SD.

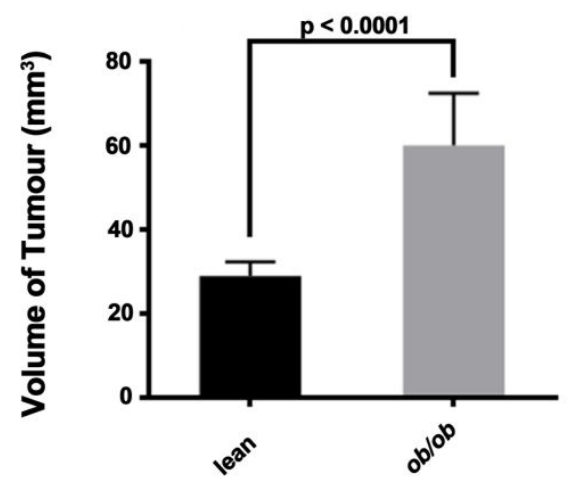


Table S1. A battery tests of systemic toxicity, including body position, grip strength, ataxic gait, tremors, skin colour and acute death. Values are systemic evaluation scores, and 0 indicates no abnormal sign.

\begin{tabular}{|c|c|c|c|c|c|c|c|c|}
\hline \multirow[b]{2}{*}{ BehaviorlAnimal group } & \multicolumn{2}{|c|}{$\begin{array}{l}\text { 0 week } \\
\end{array}$} & \multicolumn{2}{|c|}{1 week } & \multicolumn{2}{|c|}{2 week } & \multicolumn{2}{|c|}{3 week } \\
\hline & Octapod-30 & Saline & Octapod-30 & Saline & Octapod-30 & Saline & Octapod-30 & Saline \\
\hline Body position & 0 & 0 & 0 & 0 & 0 & 0 & 0 & 0 \\
\hline Touch escape & 0 & 0 & 0 & 0 & 0 & 0 & 0 & 0 \\
\hline Tail pinch & 0 & 0 & 0 & 0 & 0 & 0 & 0 & 0 \\
\hline Finger approach & 0 & 0 & 0 & 0 & 0 & 0 & 0 & 0 \\
\hline Tail elevation & 0 & 0 & 0 & 0 & 0 & 0 & 0 & 0 \\
\hline Grip strength & 0 & 0 & 0 & 0 & 0 & 0 & 0 & 0 \\
\hline Abdominal tone & 0 & 0 & 0 & 0 & 0 & 0 & 0 & 0 \\
\hline Ataxic gait & 0 & 0 & 0 & 0 & 0 & 0 & 0 & 0 \\
\hline Tremors & 0 & 0 & 0 & 0 & 0 & 0 & 0 & 0 \\
\hline Palpebral closure & 0 & 0 & 0 & 0 & 0 & 0 & 0 & 0 \\
\hline Lacrimation & 0 & 0 & 0 & 0 & 0 & 0 & 0 & 0 \\
\hline Skin color & 0 & 0 & 0 & 0 & 0 & 0 & 0 & 0 \\
\hline Acute death & 0 & 0 & 0 & 0 & 0 & 0 & 0 & 0 \\
\hline
\end{tabular}

Values are systemic evaluation scores; and, 0 indicates no abnormal sign. 
Table S2. Biochemistry analysis of mice after injection of Octapod-30 and saline for three weeks. Mouse serum hepatic enzymes of the two groups mice injected with Octapod-30 and saline with equal volume ( $n=6$ per group), including aspartate aminotransferase (AST), alanine aminotransferase (ALT), alkaline phosphatase (ALP). Data are reported as the mean $\pm \mathrm{SD}$.

\begin{tabular}{ccc}
\hline Parameter & Normal Range & Values $(n=6)$ \\
\hline AST (IU / L) & $70 \sim 120$ & $109.3 \pm 11.8$ \\
ALT (IU / L) & $\leq 45$ & $31.7 \pm 9.5$ \\
ALP (IU / L) & $30 \sim 120$ & $55.9 \pm 17.4$ \\
\hline
\end{tabular}

Values are presented as the mean \pm SD.

AST: aspartate aminotransferase

ALT: alanine aminotransferase

ALP: alkaline phosphatase 